\title{
CONHECIMENTO E PRÁTICA DOS MÉTODOS CONTRACEPTIVOS POR ESTUDANTES ADOLESCENTES: UM ESTUDO COMPARATIVO
}

\section{KNOWLEDGE AND PRACTICE OF CONTRACEPTIVE METHODS BY ADOLESCENTS STUDENTS: A COMPARATIVE STUDY}

\author{
Nytale Lindsay Cardoso Portela ${ }^{1}$ \\ Layana Pachêco de Araújo
}

RESUMO: O conhecimento sobre contracepção é imprescindível para que o jovem possa vivenciar o sexo de maneira segura. Este estudo objetivou comparar o conhecimento e a prática sobre métodos contraceptivos entre estudantes adolescentes das escolas públicas e privadas no município de Caxias, Maranhão. Trata-se de um estudo quantitativo que teve como sujeitos 231 adolescentes na faixa etária de 16 a 19 anos, sendo 32 de escolas particulares e 199, de públicas. A coleta de dados se deu pelo questionário estruturado durante os meses de agosto a outubro de 2012, sendo a análise feita com a utilização do software Epi Info 3.5.2. Dentre os adolescentes, $67,5 \%$ eram do sexo feminino e 32,5\% do masculino, com média de idade de 16,8 anos; 62,5\% dos estudantes de escolas privadas e $30,8 \%$ das públicas relataram ter iniciado a vida sexual, sendo que os homens iniciaram mais precocemente. Em relação ao uso de contraceptivos, $90,0 \%$ dos alunos de escolas particulares e $87,1 \%$ das públicas referiram utilizá-los, sendo a camisinha masculina, o método mais mencionado. No que diz respeito ao conhecimento sobre métodos contraceptivos, constatou-se que o conhecimento é insuficiente em ambas as escolas, o que pode refletir na sua prática. Reforça-se, portanto, a importância de investimentos na educação sexual dos adolescentes, sendo necessário uma articulação entre as equipes de saúde, a família e a escola para implementação de programas de educação em saúde sobre orientação sexual.

Palavras-chave: conhecimento; anticoncepção; adolescente.

ABSTRACT: Knowledge about contraception is essential so that youth may practice safe sex. This study aimed to compare knowledge and practice of contraceptive methods among students of public and private schools in the city of Caxias, Maranhão. This quantitative study had as 231 adolescents as subjects, who were 16 to 19 years old, with 32 from private and 199 from public schools. Data collection was through structured questionnaire during the months of August to October 2012, with the analysis using Epi Info 3.5.2. Of the adolescents, $67.5 \%$ were female and $32.5 \%$ male, average age 16.8 years. The study found that $62.5 \%$ of private and $30.8 \%$ of the public school students reported having already initiated sexual life, and the men started earlier. Regarding the use of contraceptives, $90.0 \%$ of private school students and $87.1 \%$ of the public reported using them, with the male condom being the most mentioned. On knowledge about contraceptive methods, it was found that the knowledge is insufficient in both schools, which may reflect in their practice. It reinforces, therefore, the importance of investment in adolescent sex education, requiring a link between health teams, family, and school to implement health education programs about sexual activity.

Keywords: knowledge; contraception; adolescent.

\footnotetext{
${ }_{1}^{1}$ Pós-graduanda em Saúde Pública e Saúde da Família - Instituto Athena. E-mail: nytalelindsay@hotmail.com.

${ }^{2}$ Mestra em Bioengenharia - Universidade do Vale do Paraíba - UNIVAP e Enfermeira da ESF da Secretaria Municipal de Saúde de Caxias - MA. E-mail: layana.pacheco@hotmail.com.
} 


\section{INTRODUÇÃO}

Segundo a Organização Mundial de Saúde (OMS), a adolescência é o período compreendido entre 10 e 19 anos de idade (WORLD HEALTH ORGANIZATION, 2002). Nessa fase, ocorre uma variedade de mudanças biopsicossociais, que trazem incertezas, questionamentos e desejos aos adolescentes, levando-os a viverem novas experiências, muitas vezes de forma impulsiva, como, por exemplo, o sexo desprotegido, que os torna vulneráveis à gravidez indesejada e precoce, além da exposição às doenças sexualmente transmissíveis (DSTs) e do vírus da imunodeficiência humana (HIV).

Cerca de 20,0\% das crianças que nascem anualmente, no Brasil, são filhos de adolescentes (SANTOS; MARSCHIN; CALDEIRA, 2007). Para Silva e Araújo (2010), a gravidez precoce tornou-se um problema de saúde pública, devido ao impacto socioeconômico e aos possíveis riscos na saúde materno-infantil. Além disso, pode acarretar no abandono da vida escolar e, até mesmo, da vida social, interferindo no desenvolvimento do indivíduo.

De acordo com Brasil (2010), foram notificados, no Sistema de Informações de Agravos de Notificação (SINAN), nos anos de 1980 a junho de 2010, 12.693 casos da síndrome da imunodeficiência adquirida (AIDS) entre adolescentes de 13 a 19 anos de idade. As DSTs e AIDS são um risco para a saúde dos adolescentes sexualmente ativos. Isso porque muitos deles possuem uma variabilidade de parceiros sexuais, têm baixa adesão ao uso de preservativo nas relações sexuais e utilizam drogas, o que facilita a transmissão de doenças (CUSTÓDIO et al., 2009).
A prevenção da gravidez e da contaminação por DST se dá por meio da utilização de métodos contraceptivos, cuja finalidade é promover relações sexuais de forma segura. Diante disso, percebe-se a necessidade da promoção da educação sexual o mais cedo possível, sendo iniciada e assumida pelos pais e complementada pela escola e equipes da Estratégia Saúde da Família.

A orientação sexual e a conscientização dos adolescentes possibilitam que estes tenham conhecimento a respeito do seu corpo e da variedade de métodos contraceptivos que podem utilizar ao iniciarem a vida sexual, sendo fundamentais para que a adolescência seja uma etapa saudável e segura.

Dado o exposto, o objetivo deste trabalho foi comparar o conhecimento e a prática sobre métodos contraceptivos entre estudantes adolescentes das escolas públicas e privadas no município de Caxias, Maranhão.

\section{MATERIAL E MÉTODOS}

Trata-se de uma pesquisa de abordagem quantitativa e caráter descritivo, realizada no período de agosto a outubro de 2012, em escolas de Ensino Médio da zona urbana de Caxias, Maranhão. Fizeram parte do estudo duas escolas públicas e duas particulares, escolhidas de forma aleatória por meio de sorteio.

O tamanho da amostra foi calculado a partir da população de 35 estudantes adolescentes do Ensino Médio das escolas particulares, sendo 16 da primeira escola sorteada e 19, da segunda; e 396 adolescentes das escolas públicas (185 da 
primeira escola e 211, da segunda). Utilizando-se o cálculo do tamanho da amostra de Barbetta (2006) e considerando a margem de erro tolerável de $5 \%$ e nível de confiança de $95 \%$ na população de cada escola, obteve-se uma população de 231 adolescentes, dos quais 32 estudavam em escolas particulares e 199 nas instituições públicas.

A pesquisa foi realizada após 0 consentimento do Gestor da Unidade Regional de Educação de Caxias-MA; autorização da pesquisa pelo Comitê de Ética em Pesquisa da Universidade Estadual do Maranhão, sob o no de protocolo 07898512.2.0000.5554 e permissão dos Diretores das Escolas selecionadas, seguido do aceite do estudante adolescente de participar, voluntariamente, do estudo e da assinatura do Termo de Consentimento Livre e Esclarecido (TCLE) pelo responsável, no caso de adolescentes menores de idade, ou pelo próprio adolescente, para aqueles com 18 anos ou mais.

A população do estudo foi constituída por adolescentes na faixa etária de 16 a 19 anos, pois somente os alunos nessa faixa etária apresentaram o TCLE assinado, permitindo, assim, sua participação na pesquisa.

Utilizou-se de um questionário estruturado, autopreenchível e anônimo, contendo questões acerca das características sociodemográficas, características sexuais e conhecimento sobre métodos contraceptivos. A elaboração do questionário baseou-se no Manual lançado em 2009 de "Direitos sexuais, direitos reprodutivos e métodos anticoncepcionais", do Ministério da Saúde.

A análise dos dados foi feita com a utilização do software Epi Info (version 3.5.2; 2010), em um contexto quantitativo, expressos mediante símbolos numéricos. A análise estatística foi descritiva, sendo os dados organizados em forma de tabelas para análise e discussão.

\section{RESULTADOS E DISCUSSÃO}

A Tabela 1 apresenta a população de adolescentes estudados com base nas características sociodemográficas e sexuais.

A população do estudo foi constituída de 231 adolescentes pesquisados, distribuídos em 156 mulheres (67,5\%) e 75 homens $(32,5 \%)$, havendo predomínio do sexo feminino tanto nas escolas particulares quanto nas públicas (53,1\% vs $69,8 \%$ ). As idades dos adolescentes estudados variaram de 16 a 19 anos, sendo a média de idade de 17,0 anos nas escolas particulares e 16,7 anos, nas públicas.

Em relação à cor da pele, $56,3 \%$ dos entrevistados das escolas particulares e $61,3 \%$ das públicas se autodefiniram como pardos. Quanto à religião referida, houve predomínio da religião católica em ambas as escolas $(62,5 \%$ nas particulares vs $66,3 \%$ nas públicas), seguida da evangélica $(21,9 \%$ nas particulares vs $22,6 \%$ nas públicas). Houve, também, aqueles que relataram não ter nenhuma religião, totalizando $10,4 \%$ dos entrevistados. 
Tabela 1 - Número e distribuição percentual das características sociodemográficas e sexuais dos adolescentes segundo o tipo de escola. Caxias, Maranhão, 2012

\begin{tabular}{|c|c|c|c|c|c|c|}
\hline \multirow{3}{*}{$\begin{array}{c}\text { CARACTERÍSTICAS } \\
\text { SOCIODEMOGRÁFICAS E SEXUAIS }\end{array}$} & \multicolumn{4}{|c|}{ TIPO DE ESCOLA } & \multirow{2}{*}{\multicolumn{2}{|c|}{ TOTAL }} \\
\hline & \multicolumn{2}{|c|}{ PARTICULAR } & \multicolumn{2}{|c|}{ PÚBLICA } & & \\
\hline & $\mathbf{N}$ & $\%$ & $\mathbf{N}$ & $\%$ & $\mathbf{N}$ & $\%$ \\
\hline \multicolumn{7}{|l|}{ Idade } \\
\hline 16 anos & 11 & 34,4 & 102 & 51,3 & 113 & 48,9 \\
\hline 17 anos & 11 & 34,4 & 66 & 33,2 & 77 & 33,3 \\
\hline 18 anos & 08 & 25,0 & 19 & 9,5 & 27 & 11,7 \\
\hline 19 anos & 02 & 6,3 & 12 & 6,0 & 14 & 6,1 \\
\hline \multicolumn{7}{|l|}{ Sexo } \\
\hline Feminino & 17 & 53,1 & 139 & 69,8 & 156 & 67,5 \\
\hline Masculino & 15 & 46,9 & 60 & 30,2 & 75 & 32,5 \\
\hline \multicolumn{7}{|l|}{ Cor } \\
\hline Branca & 05 & 15,6 & 19 & 9,5 & 24 & 10,4 \\
\hline Parda & 18 & 56,3 & 122 & 61,3 & 140 & 60,6 \\
\hline Negra & 05 & 15,6 & 48 & 24,1 & 53 & 22,9 \\
\hline Amarela & 03 & 9,4 & 08 & 4,0 & 11 & 4,8 \\
\hline Indígena & 01 & 3,1 & 02 & 1,0 & 03 & 1,3 \\
\hline Outra & 00 & 0,0 & 00 & 0,0 & 00 & 0,0 \\
\hline \multicolumn{7}{|l|}{ Religião } \\
\hline Católica & 20 & 62,5 & 132 & 66,3 & 152 & 65,8 \\
\hline Protestante/Evangélica & 07 & 21,9 & 45 & 22,6 & 52 & 22,5 \\
\hline Espírita & 00 & 0,0 & 00 & 0,0 & 00 & 0,0 \\
\hline Outra & 00 & 0,0 & 01 & 0,5 & 01 & 0,4 \\
\hline Não tenho religião, mas acredito em Deus & 04 & 12,5 & 20 & 10,1 & 24 & 10,4 \\
\hline Não acredito em Deus & 01 & 3,1 & 01 & 0,5 & 02 & 0,9 \\
\hline \multicolumn{7}{|l|}{ Estado Civil } \\
\hline Solteiro(a) & 30 & 93,8 & 190 & 95,5 & 220 & 95,2 \\
\hline Casado(a) & 00 & 0,0 & 04 & 2,0 & 04 & 1,7 \\
\hline Vive/mora junto com um(a) companheiro(a) & 02 & 6,3 & 05 & 2,5 & 07 & 3,0 \\
\hline Separado(a)/Divorciado(a) & 00 & 0,0 & 00 & 0,0 & 00 & 0,0 \\
\hline Viúvo(a) & 00 & 0,0 & 00 & 0,0 & 00 & 0,0 \\
\hline \multicolumn{7}{|l|}{ Renda Familiar } \\
\hline Sem renda & 00 & 0,0 & 14 & 7,0 & 14 & 6,1 \\
\hline Até um salário-mínimo & 04 & 12,5 & 76 & 38,2 & 80 & 34,6 \\
\hline Mais de 1 até 3 salários-mínimos & 09 & 28,1 & 81 & 40,7 & 90 & 39,0 \\
\hline Mais de 3 até 5 salários-mínimos & 14 & 43,8 & 23 & 11,6 & 37 & 16,0 \\
\hline Mais de 5 até 10 salários-mínimos & 03 & 9,4 & 03 & 1,5 & 06 & 2,6 \\
\hline Mais de 10 salários-mínimos & 02 & 6,3 & 02 & 1,0 & 04 & 1,7 \\
\hline \multicolumn{7}{|l|}{ Relação Sexual } \\
\hline Sim & 20 & $62,5 \%$ & 62 & $31,2 \%$ & 82 & $35,5 \%$ \\
\hline Não & 12 & $37,5 \%$ & 137 & $68,8 \%$ & 149 & $64,5 \%$ \\
\hline TOTAL & $\begin{array}{c}32 \\
13,9 \\
\end{array}$ & 100,0 & $\begin{array}{l}199 \\
86,1\end{array}$ & 100,0 & 231 & 100,0 \\
\hline
\end{tabular}

No que diz respeito ao estado civil, a maior parte dos adolescentes referiu ser solteira, totalizando 220 indivíduos, sendo $30 \quad(93,8 \%)$ adolescentes das escolas particulares e 190 (95,5\%) das públicas.
Entre os 11 adolescentes que relataram já ter sido unido alguma vez, 4 estavam casados $(2,0 \%$ dos adolescentes das escolas públicas) e 7 viviam unidos consensualmente, sendo 2 das escolas 
privadas $(6,3 \%)$ e 5 das públicas $(2,5 \%)$.

Em relação à renda familiar mensal, prevaleceu, nas escolas privadas, renda mensal de 3 a 5 salários mínimos (43,8\%), seguida da renda de 1 a 3 salários mínimos (23,1\%). Nas escolas públicas, $40,7 \%$ dos adolescentes referiram ter uma renda familiar de 1 a 3 salários mínimos; 38,2\% têm até um salário mínimo e 7,0\% não possuem renda.

Quanto às características sexuais dos adolescentes, $64,5 \%$ referiram não ter iniciado a vida sexual. No entanto, esse resultado não é equivalente nas escolas públicas e particulares. Observa-se que, nas escolas privadas, 62,5\% dos adolescentes relataram ter tido relação sexual. Já, nas escolas públicas, apenas 30,8\% da população, deram início à vida sexual.

Os dados encontrados corroboram com um estudo desenvolvido por Martins et al. (2006), no qual se verificou que dos 1.594 adolescentes entre 12 e 19 anos, do município de São Paulo, 81,4\% e 71,4\% dos alunos das escolas particulares e públicas, respectivamente, não iniciaram essa prática.

Portanto, mesmo com a iniciação da vida sexual em idades cada vez mais precoces, observa-se que a maioria dos adolescentes ainda não teve a primeira relação sexual. O percentual de jovens que já tiveram relação sexual foi maior nas escolas particulares do que nas públicas, provavelmente porque os garotos iniciam a vida sexual mais cedo e, no estudo, o número de jovens do sexo masculino e feminino nas escolas privadas quase se iguala, enquanto que, nas instituições públicas, a quantidade de mulheres é 2,3 vezes maior do que a de homens.

Além disso, pode ter tido algum tipo de viés, pois, respondendo às expectativas sociais e com o receio de que os outros participantes estivessem os observando, os homens podem ter referido já ter tido relação sexual e, em contrapartida, em relação às mulheres, pode ter ocorrido o oposto. Ou ainda, considerando que as características de um grupo ou comunidade interferem no comportamento sexual, o início da vida sexual pode ter sido diferente devido às populações estudadas serem distintas.

$\mathrm{Na}$ Tabela 2, observa-se que a primeira relação sexual dos adolescentes do sexo masculino ocorreu entre 10 a 18 anos de idade, tendo mediana de 14 anos nos estudantes de escolas particulares e mediana de 15 anos, nos de escolas públicas. Nestas, dois dos entrevistados não responderam quando questionados sobre a idade na primeira relação sexual.

Nas adolescentes do sexo feminino, quase não houve diferença de idade da primeira relação sexual entre as estudantes de escola privadas e públicas, sendo mediana de 15 anos e 16 anos, respectivamente. Nas escolas públicas, quatro das entrevistadas não responderam tal questionamento.

Assim, considerando a medida de tendência central (mediana), observa-se que os homens iniciaram a vida sexual um ano antes do que as mulheres, revelando que a média de idade dos garotos, na primeira relação sexual, é menor que nas garotas. 
Tabela 2 - Número e percentual de adolescentes que iniciaram a vida sexual, segundo idade na primeira relação sexual, sexo e tipo de escola. Caxias, Maranhão, 2012

\begin{tabular}{|c|c|c|c|c|c|c|}
\hline \multirow{3}{*}{$\begin{array}{l}\text { IDADE NA PRIMEIRA RELAÇÃO } \\
\text { SEXUAL }\end{array}$} & \multicolumn{4}{|c|}{ TIPO DE ESCOLA } & \multirow{2}{*}{\multicolumn{2}{|c|}{ TOTAL }} \\
\hline & \multicolumn{2}{|c|}{ PARTICULAR } & \multicolumn{2}{|c|}{ PÚBLICA } & & \\
\hline & $\mathbf{N}$ & $\%$ & N & $\%$ & $\mathbf{N}$ & $\%$ \\
\hline \multicolumn{7}{|l|}{ Sexo Masculino } \\
\hline 10 anos & 01 & 8,3 & 00 & 0,0 & 01 & 2,6 \\
\hline 12 anos & 01 & 8,3 & 01 & 3,7 & 02 & 5,1 \\
\hline 13 anos & 01 & 8,3 & 03 & 11,1 & 04 & 10,3 \\
\hline 14 anos & 06 & 50,0 & 05 & 18,5 & 11 & 28,2 \\
\hline 15 anos & 03 & 25,0 & 06 & 22,2 & 09 & 23,1 \\
\hline 16 anos & 00 & 0,0 & 08 & 29,6 & 08 & 20,5 \\
\hline 17 anos & 00 & 0,0 & 01 & 3,7 & 01 & 2,6 \\
\hline 18 anos & 00 & 0,0 & 01 & 3,7 & 01 & 2,6 \\
\hline Sem resposta & 00 & 0,0 & 02 & 7,4 & 02 & 5,1 \\
\hline Subtotal & 12 & 100,0 & 27 & 100,0 & 39 & 100,0 \\
\hline Média & 13,7 & & 15,0 & & 14,5 & \\
\hline Mediana & 14 & & 15 & & 15 & \\
\hline \multicolumn{7}{|l|}{ Sexo Feminino } \\
\hline 08 anos & 00 & 0,0 & 01 & 2,9 & 01 & 2,3 \\
\hline 13 anos & 00 & 0,0 & 03 & 8,6 & 03 & 7,0 \\
\hline 14 anos & 03 & 37,5 & 02 & 5,7 & 05 & 11,6 \\
\hline 15 anos & 02 & 25,0 & 07 & 20,0 & 09 & 20,9 \\
\hline 16 anos & 02 & 25,0 & 12 & 34,3 & 14 & 32,6 \\
\hline 17 anos & 00 & 0,0 & 06 & 17,1 & 06 & 13,9 \\
\hline 18 anos & 01 & 12,5 & 00 & 0,0 & 01 & 2,3 \\
\hline Sem resposta & 00 & 0,0 & 04 & 11,4 & 04 & 9,3 \\
\hline Subtotal & 8 & 100,0 & 35 & 100,0 & 43 & 100,0 \\
\hline Média & 15,2 & & 15,3 & & 15,3 & \\
\hline Mediana & 15 & & 16 & & 16 & \\
\hline TOTAL & $\begin{array}{c}20 \\
24,4\end{array}$ & 100,0 & $\begin{array}{c}62 \\
75,6\end{array}$ & 100,0 & 82 & 100,0 \\
\hline
\end{tabular}

A média de idade na primeira relação sexual para os estudantes do sexo masculino (14,5 anos) e feminino (15,3 anos) do presente estudo foi semelhante à verificada na pesquisa realizada com escolares do ensino fundamental e médio de 13 capitais brasileiras e do Distrito Federal, com idade entre 10 e 24 anos, por Abramovay, Castro e Silva (2004), na qual a média de idade variou de 13,4 a 14,5 anos para os homens e de 15,0 a 16,0 anos para as mulheres.

Em relação à idade no início da vida sexual, surpreendentemente encontraram-se declarações de iniciação sexual a partir dos oito anos de idade entre as mulheres. Entre os homens, por outro lado, entende-se ter sido relatado que aos dez anos ocorreu a primeira experiência sexual, pois o jovem do sexo masculino sente a necessidade de comprovar a sua masculinidade para a sociedade. 
Cabe ressaltar que a coleta de dados foi realizada em sala de aula, o que pode ter feito com que os participantes se sentissem observados pelos colegas, podendo ter sido levados a exagerar na indicação da idade da primeira relação sexual.

Tabela 3 - Distribuição percentual dos adolescentes que já apresentaram relação sexual, segundo o tipo de escola e o uso de método contraceptivo. Caxias, Maranhão, 2012

\begin{tabular}{|c|c|c|c|}
\hline \multirow[b]{2}{*}{$\begin{array}{l}\text { USO DE MÉTODO } \\
\text { CONTRACEPTIVO }\end{array}$} & \multicolumn{2}{|c|}{ TIPO DE ESCOLA } & \multirow[b]{2}{*}{ TOTAL } \\
\hline & PARTICULAR & PÚBLICA & \\
\hline $\begin{array}{l}\text { Sim } \\
\text { Não }\end{array}$ & $\begin{array}{l}90,0 \% \\
10,0 \%\end{array}$ & $\begin{array}{l}87,1 \% \\
12,9 \%\end{array}$ & $\begin{array}{l}87,8 \% \\
12,2 \%\end{array}$ \\
\hline
\end{tabular}

Na Tabela 3, percebe-se que, apesar de haver uma considerável parcela de alunos que utilizam métodos contraceptivos $(87,8 \%)$, ainda existe um número razoável de adolescentes que tem relação e não utiliza nenhuma forma de contracepção $(12,2 \%)$.

Boruchovitch (1992), estudando os fatores associados a não utilização de métodos contraceptivos na adolescência, constatou que a falta de conhecimento do adolescente acerca de questões sexuais, a má informação acerca dos métodos existentes, o pensamento de que 0 contraceptivo interfere no prazer sexual, a baixa autoestima, a percepção de invulnerabilidade causam uma menor procura e uso dos métodos contraceptivos.

Concorda-se com Boruchovitch (1992), quando se observa na Tabela 5, onde $9,4 \%$ dos adolescentes das escolas particulares e $4,5 \%$ das públicas referiram à falta de informação acerca dos métodos contraceptivos existentes. Tal fato leva ao conhecimento incorreto do seu uso, como o pensamento de que o DIU interfere no prazer sexual, de que o coito interrompido é eficaz na prevenção de gravidez e de que a pílula anticoncepcional de emergência é eficaz na prevenção da gravidez, mesmo sendo utilizada mais de uma vez.

$$
\text { Conforme a Tabela 4, ao serem }
$$
questionados sobre os métodos contraceptivos que utilizavam nas relações sexuais, a camisinha masculina foi a mais citada entre os adolescentes dos sexos masculino e feminino, tanto nas escolas particulares $(83,3 \%$ dos garotos e $50,0 \%$ das garotas) quanto nas públicas $(91,3 \%$, masculino e, 67,7\%, feminino).

Tal resultado também foi encontrado por Almeida et al. (2003), que, ao estudarem o comportamento contraceptivo de 1.664 adolescentes de oito escolas públicas do estado da Bahia, constataram que tanto os rapazes quanto as moças utilizaram o preservativo masculino tanto na primeira (90,1\%, sexo masculino e, $73,5 \%$, feminino) quanto na última relação sexual $(60,3 \%$ dos homens e $43,2 \%$ das mulheres). 
Tabela 4 - Métodos contraceptivos utilizados por adolescentes, segundo sexo e tipo de escola. Caxias, Maranhão, 2012

\begin{tabular}{|c|c|c|c|c|c|c|}
\hline \multirow{3}{*}{ MÉTODOS CONTRACEPTIVOS } & \multicolumn{4}{|c|}{ TIPO DE ESCOLA } & \multirow{2}{*}{\multicolumn{2}{|c|}{ TOTAL }} \\
\hline & \multicolumn{2}{|c|}{ PARTICULAR } & \multicolumn{2}{|c|}{ PÚBLICA } & & \\
\hline & & $\%$ & $\mathrm{~N}$ & $\%$ & $\mathbf{N}$ & $\%$ \\
\hline \multicolumn{7}{|l|}{ Sexo Masculino } \\
\hline Camisinha Masculina (CM) & 10 & 83,3 & 21 & 91,3 & 31 & 88,6 \\
\hline $\mathrm{CM}+$ Anticoncepcional Oral Combinado & 02 & 16,7 & 00 & 0,0 & 02 & 5,7 \\
\hline Sem resposta & 00 & 0,0 & 02 & 8,7 & 02 & 5,7 \\
\hline Subtotal & 12 & 100,0 & 23 & 100,0 & 35 & 100,0 \\
\hline \multicolumn{7}{|l|}{ Sexo Feminino } \\
\hline Camisinha Masculina (CM) & 03 & 50,0 & 21 & 67,7 & 24 & 64,9 \\
\hline Anticoncepcional Oral Combinado (ACO) & 01 & 16,7 & 02 & 6,4 & 03 & 8,1 \\
\hline $\mathrm{CM}+\mathrm{ACO}$ & 00 & 0,0 & 03 & 9,7 & 03 & 8,1 \\
\hline $\mathrm{CM}+$ Anticoncepção de Emergência (AE) & 00 & 0,0 & 01 & 3,2 & 01 & 2,7 \\
\hline $\mathrm{CM}+$ Anticoncepcional Injetável (Al) & 00 & 0,0 & 01 & 3,2 & 01 & 2,7 \\
\hline $\mathrm{CM}+\mathrm{ACO}+\mathrm{AE}$ & 01 & 16,7 & 01 & 3,2 & 02 & 5,4 \\
\hline $\mathrm{CM}+\mathrm{Al}+\mathrm{AE}$ & 00 & 0,0 & 01 & 3,2 & 01 & 2,7 \\
\hline Sem resposta & 01 & 16,7 & 01 & 3,2 & 02 & 5,4 \\
\hline Subtotal & 06 & 100,0 & 31 & 100,0 & 37 & 100,0 \\
\hline TOTAL & $\begin{array}{c}18 \\
25,0\end{array}$ & 100,0 & $\begin{array}{c}54 \\
75,0\end{array}$ & 100,0 & 72 & 100,0 \\
\hline
\end{tabular}

A maioria dos adolescentes relatou utilizar a camisinha masculina como método contraceptivo. Tal fato pode ocorrer devido à divulgação em massa desse método e o desconhecimento dos diversos tipos de contraceptivos existentes. Além disso, alguns podem ter referido 0 uso do preservativo masculino por saberem que este é de suma importância durante a relação sexual para se prevenir de agravos.

De acordo com a Tabela 5, quando verificada a presença de informação sobre métodos contraceptivos, constatou-se que, nas escolas particulares, $90,6 \%$ dos estudantes referiram já ter recebido informações sobre 0 assunto $e$, nas públicas, observa-se que $95,5 \%$ dos estudantes receberam informações.
Como foi observado na Tabela 3 , o primeiro contato sexual está ocorrendo cada vez mais cedo entre os jovens. Garotos e garotas estão se tornando sexualmente ativos em um período de dúvidas sobre o corpo, a identidade, a sexualidade, entre outros temas, o que pode levá-los a adquirirem problemas, como gravidez não planejada, DSTs e uso de drogas. Com isso, percebe-se a importância da orientação sexual a partir do instante que o adolescente começa a manifestar as alterações biopsicossociais dessa fase. No entanto, alguns adolescentes, o equivalente a $5,2 \%$ da população estudada, nunca tiveram informações sobre os métodos contraceptivos, como pode ser observado na Tabela 5. 
Tabela 5 - Distribuição percentual dos adolescentes, segundo presença de informação sobre métodos contraceptivos e o tipo de escola. Caxias, Maranhão, 2012

\begin{tabular}{|c|c|c|c|}
\hline \multirow[b]{2}{*}{$\begin{array}{c}\text { INFORMAÇÃO SOBRE } \\
\text { MÉTODOS } \\
\text { CONTRACEPTIVOS }\end{array}$} & \multicolumn{2}{|c|}{ TIPO DE ESCOLA } & \multirow[b]{2}{*}{$\begin{array}{c}\text { TOTAL } \\
\text { (\%) }\end{array}$} \\
\hline & $\begin{array}{c}\text { PARTICULAR } \\
\text { (\%) }\end{array}$ & $\begin{array}{l}\text { PÚBLICA } \\
(\%)\end{array}$ & \\
\hline Sim & $90,6 \%$ & $95,5 \%$ & $94,8 \%$ \\
\hline Não & $9,4 \%$ & $4,5 \%$ & $5,2 \%$ \\
\hline
\end{tabular}

Na Tabela 6, que trata da proporção de adolescentes que respondeu corretamente sobre métodos contraceptivos, observa-se que a injeção anticoncepcional e a camisinha masculina foram os métodos contraceptivos de maior índice de acerto nas escolas particulares e públicas.

Nas escolas particulares, a questão que os adolescentes mais acertaram foi a referente à camisinha masculina, com $65,6 \%$, seguida das de diafragma e dispositivo intrauterino (56,3\%), e tabelinha $(50,0 \%)$. Nas escolas públicas, os maiores números de acertos foram sobre injeção anticoncepcional (64,3\%), camisinha masculina $(53,8 \%)$, dispositivo intrauterino $(51,0 \%)$ e coito interrompido (50,3\%). Observa-se que nessas questões, pelo menos, metade dos alunos marcaram as alternativas corretas.

Martins et al. (2006), analisando o conhecimento sobre métodos contraceptivos de 1.594 adolescentes de escolas públicas e privadas do município de São Paulo-SP, constataram que a questão sobre camisinha feminina foi a que teve maior índice de acerto nos dois grupos de escolas (88,4\% nas privadas e $84,6 \%$ nas públicas). Além disso, observaram, também, que os estudantes tinham conhecimento adequado sobre camisinha masculina, cujo índice de acerto foi superior a $70,0 \%$, tanto nas escolas públicas, quanto nas particulares. Tais resultados são diferentes dos encontrados no presente estudo.

Nota-se, ainda, na Tabela 6, que, nas escolas privadas, algumas questões tiveram maior número de erros do que acertos. Tais questões tratavam de pílula anticoncepcional, pílula anticoncepcional de emergência, camisinha feminina e muco cervical. Nas escolas públicas, tal fato foi observado nas questões referentes à pílula anticoncepcional de emergência e camisinha feminina.

Faz-se, no entanto, uma ressalva em relação às pílulas anticoncepcionais de emergência. Alguns autores, como Silva (2003), Eisenstein, Bouzas e Pacheco (2005), Cordellini et al. (2006) e Poli et al. (2009), consideram que a contracepção de emergência deve ser utilizada em até três dias. Outros, porém, afirmam que a mesma pode ser usada até cinco dias após o coito (LEAL; SAITO, 2007; BRASIL, 2009). Cabe aqui, ressaltar que, nas palestras realizadas com esses jovens, geralmente é dito que a pílula anticoncepcional de emergência deve ser utilizada em 72 horas após a relação sexual. Portanto, pode-se considerar que os adolescentes que responderam que a pergunta estava correta e àqueles que a marcaram como incorreta acertaram a questão. 
Tabela 6 - Porcentagem de respostas corretas sobre métodos contraceptivos, segundo o tipo de escola. Caxias, Maranhão, 2012

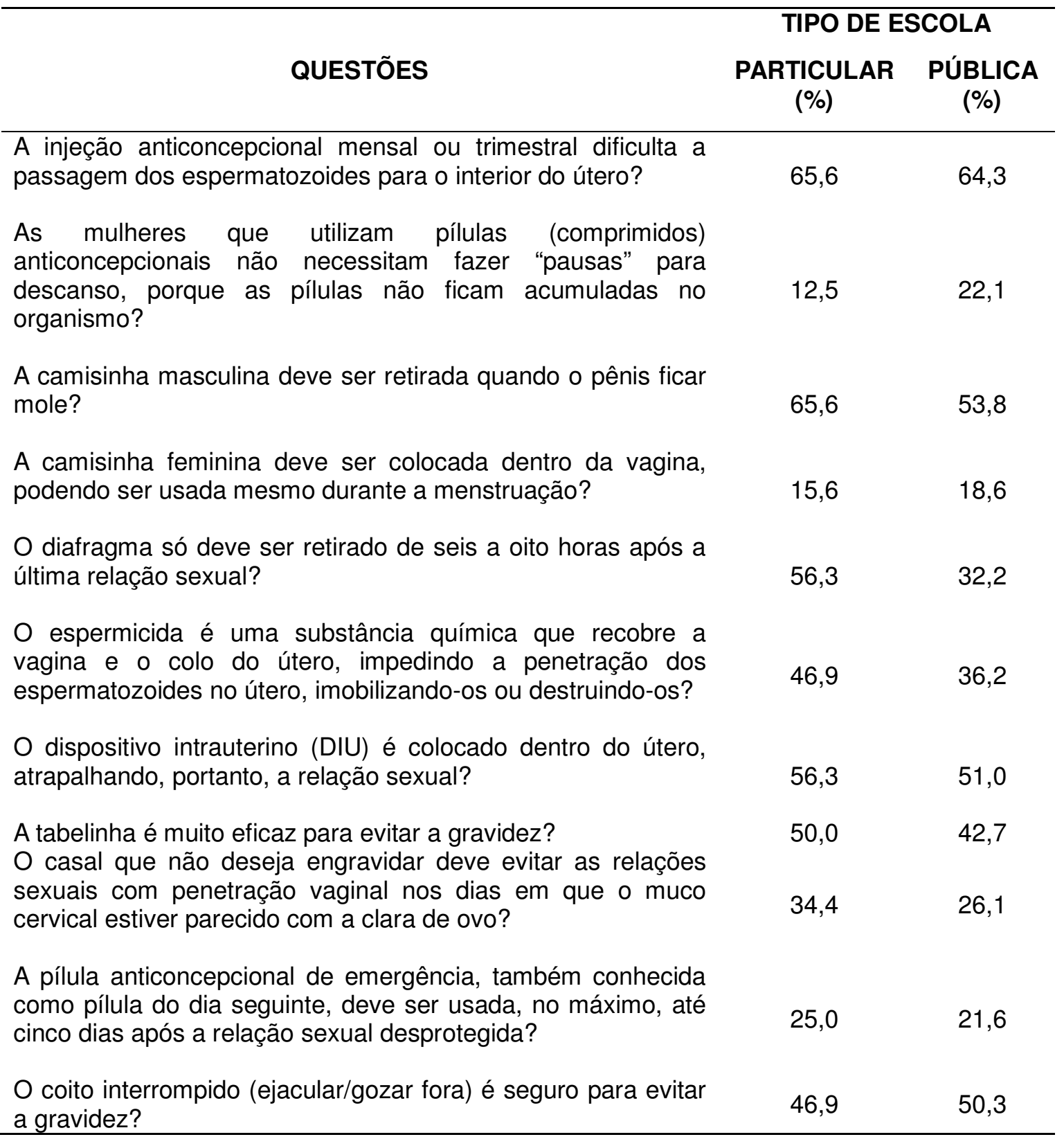

Embora os estudantes de escolas particulares tenham apresentado conhecimento um pouco maior que os adolescentes das escolas públicas, 0 conhecimento, em ambas as escolas, é insatisfatório, considerando que nas questões com maior número de acertos, constatou-se apenas $65,6 \%$ e $64,3 \%$ de respostas corretas dos alunos de escolas privadas e públicas, respectivamente. Isso sugere que, mesmo a maioria tendo respondido que utilizam métodos contraceptivos nas relações sexuais, eles estão com pouca informação acerca do assunto, o que os coloca em risco de ter uma gravidez precoce e indesejada ou adquirir uma DST. 


\section{CONCLUSÃO}

O presente estudo, ao analisar duas amostras estudantis provenientes de redes de ensino distintas, permitiu comparar a prática sexual dos adolescentes e o conhecimento sobre métodos contraceptivos em diferentes condições socioeconômicas.

Os resultados mostraram que $62,5 \%$ dos estudantes das escolas privadas e $31,2 \%$ das públicas já haviam iniciado a vida sexual e que os adolescentes do sexo masculino têm a primeira relação sexual com maior precocidade do que as do sexo feminino. Dos adolescentes sexualmente ativos, o equivalente a $90,0 \%$ dos alunos das escolas privadas e $87,1 \%$ das públicas utilizavam algum método contraceptivo nas relações sexuais, sendo a camisinha masculina o método mais referido.

Apesar da utilização de contraceptivos pela maioria dos entrevistados e de estes terem sido informados de alguma forma sobre contracepção, constatou-se que os estudantes, tanto das escolas privadas quando das públicas, não têm conhecimento suficiente acerca do assunto, o que sugere que adolescentes não estão tendo informações de boa qualidade, não conhecendo, portanto, os vários métodos existentes, como se utiliza e suas vantagens e desvantagens.

Os presentes resultados reforçam a importância de investimentos na educação sexual dos adolescentes em geral. É necessária uma articulação entre as equipes de saúde, a família e a escola para implementação de programas de educação em saúde sobre orientação sexual.

Somente com a orientação e conscientização dos adolescentes, acerca da importância da utilização de métodos contraceptivos durante as relações sexuais, é possível diminuir os índices de gravidez precoce e DST/AIDS, contribuindo, assim, para a saúde e o bem-estar biopsicossocial dessa parcela da população.

\section{REFERÊNCIAS}

ABRAMOVAY, M.; CASTRO, M. G.; SILVA, L. B. Juventudes e Sexualidade. Brasília: UNESCO Brasil, 2004.

ALMEIDA, M. C. C. et al. Uso de contracepção por adolescentes de escolas públicas na Bahia. Revista Saúde Pública, São Paulo, v. 37, n. 5, p. 566-575, 2003.

BARBETTA, P. A. Estatística aplicada às ciências sociais. 6. ed. Florianópolis: UFSC, 2006.

BORUCHOVITCH, E. Fatores associados a não utilização de anticoncepcionais na adolescência. Revista de Saúde Pública, São Paulo, v. 26, n. 6, p. 437-443, 1992.

BRASIL. Ministério da Saúde. Boletim Epidemiológico - AIDS e DST. Ano VII no 1 . Brasília (DF): Ministério da Saúde Secretaria de Vigilância em Saúde Departamento de DST, AIDS e Hepatites Virais, 2010. . Ministério da Saúde. Secretaria de Atenção à Saúde. Departamento de Ações Programáticas Estratégicas. Direitos sexuais, direitos reprodutivos e métodos anticoncepcionais. Brasília: Ministério da Saúde, 2009. 52p.

CORDELLINI, J. V. F. et al. Protocolo de atenção à saúde do adolescente. 2. ed. rev. e atual. Curitiba: Secretaria Municipal de Saúde, 2006.

CUSTÓDIO, G. et al. Comportamento sexual e de risco para DST e gravidez em 
adolescentes. DST - Jornal Brasileiro de Doenças Sexualmente Transmissíveis, Rio de Janeiro, v. 21, n. 2, p. 60-64, 2009.

EISENSTEIN, E.; BOUZAS, I.; PACHECO, A. Orientação Contraceptiva na Adolescência. In: Sociedade de Pediatria do Rio Grande do Sul - SPRS. Pediatria: diagnóstico e tratamento. Porto Alegre: Artmed, 2005. p. 399-408.

LEAL, M. M.; SAITO, M. I. Anticoncepção e Adolescência. In: Sociedade Brasileira de Pediatria. Tratado de Pediatria. Barueri, SP: Manole, 2007. cap. 7, p. 405-415.

MARTINS, L. B. M. et al. Conhecimento sobre os métodos anticoncepcionais por estudantes adolescentes. Revista de Saúde Pública, São Paulo, v. 40, n. 1, p. 57-64, 2006.

POLI, M. E. H. et al. Manual de anticoncepção da FEBRASGO. FEMINA, v. 37, n. 9, p. 459-492, set. 2009.
SANTOS, D. R.; MARSCHIN, M. S.; CALDEIRA, S. Percepção dos enfermeiros frente à gravidez na adolescência. Ciência, Cuidado e Saúde, Paraná, v. 6, n. 4, p. 479485, 2007.

SILVA, J. L. C. P. Anticoncepção na Adolescência. In: MARCONDES, Eduardo; VAZ, Flávio Adolfo Costa; RAMOS, José Lauro Araújo; OKAY, Yassuhiko. Pediatria Básica: Tomo I - Pediatria Geral e Neonatal. 9. ed. São Paulo: SARVIER, 2003. Sétima Parte. cap. 11, p. 693-697.

SILVA, L. F.; ARAÚJO, L. P. Conhecimento e adesão de mães adolescentes acerca do planejamento familiar. 2010. 87 p. Monografia (Bacharelado em Enfermagem) Universidade Estadual do Maranhão, Caxias-MA, 2010.

WHO (WORLD HEALTH ORGANIZATION). Adolescent Friendly Health Services - An Agenda for Change. Geneva: WHO, 2002. 